\title{
A Histogram Equalization Algorithm about Second Generation Wavelet Integer Transform
}

\author{
Jianye Peng ${ }^{1, a}$, Yi Zhang ${ }^{1}$ \\ ${ }^{1}$ Information Center, Jiangsu University of Technology, Changzhou, Jiangsu Province, China \\ apengjianye@jsut.edu.cn
}

Keywords: histogram equalization; second generation wavelet; wavelet coefficient; luminance maintenance.

\begin{abstract}
In view of the traditional processing defects of histogram image, this paper proposes a new histogram equalization algorithm about second generation wavelet integer transform. Firstly, the method of three level lifting was used to improve to the wavelet integer transform and to extract the low frequency coefficients. Secondly, the low frequency wavelet coefficients are divided into four sub images with the help of the average luminance wavelet coefficient. Each sub image was extracted by histogram equalization. Finally, the four sub images were merged in order to construct an enhanced image. The experiment results showed that the algorithm of this paper is better in image luminance maintenance and noise controlling and has clearer details. The visual effects of the enhanced images can be applied in remote sensing satellite and the processing of medical images, etc.
\end{abstract}

\section{Introduction}

There are many algorithms enhancing the images. The histogram equalization is much more classic and effective. Despite the obvious advantages of fast arithmetic speed and great enhancement effectiveness, it still has the following shortcomings: (1) The histogram equalization would cause poor layering sense because of the small dynamic range of the original image' s gray scale, its poor quality and uneven histogram equalization. (2) After the histogram equalization, the noise in the original is obviously enlarged. (3) If the image gray scale is close to 0 , during the histogram equalization, a brighter diluted image would be output when the very narrow range of dark pixels is mapped to the output image. This would affect the basic features of the image (for instance, the average luminance would be changed; the details would be lost), which would finally influence the visual effects to enhance the image and make the applications of histogram algorithm limited.

The multi-scale analysis of wavelet can be used to provide more flexible processing methods, and the optimal results can be obtained by using the least amount of computation. In this paper, we use wavelet transform to balance the low frequency coefficients of the three layer decomposition of images, and then we research on the enhancement algorithm for the reconstruction of the equalized sub band coefficients.

\section{The Enhancement Algorithm of Histogram Equalization about Second Generation Wavelet Integer Transform}

To improve wavelet. In the process of wavelet transform, the filter output of traditional wavelet transform is floating point number while the pixel value of the image is integer. The wavelet lifting scheme is proposed to construct a new view; that is, lifting scheme. It is also known as the second generation wavelet integer transform. The wavelet lifting scheme has a real sense of reversibility, which can ignore the boundary effect. Compared with the traditional wavelet transform, the proposed scheme has the following advantages: a) It inherits the multi-resolution feature of the first generation wavelet. The quality of output image would not be influenced by the serial length. It can also transform the arbitrary size images. b) The construction of wavelet is within the spatial domain. It can be without Fourier Analysis Theory. c) The involved instruments are very easy, mainly including Euclidean division with Laurent series. All the traditional wavelet can be improved and divided by 
this lifting scheme. d) The arithmetic speed is fast, which helps to save storing space. e) It can realize transform among integers, which finally make the lossless compression which is based on wavelet come true.

Since the wavelet of the image is completely reversible, the symmetrical structures and opposite operations can realize the exact reconstruction.

Algorithm description. The traditional convolution based on discrete wavelet transform is of great computational complexity, which requires a large storage space. Therefore, it is not conducive for hardware implementation. However, the wavelet lifting algorithm has the advantages of simple structure, low computation and can save storage space. It can also realize the reversible transform from integer to integer. All these features help to realize hardware implementation. Therefore, the algorithm uses second generation wavelet integer lifting algorithm, that is, the second generation wavelet integer transform. The image is decomposed into three layers. Before the third layer low frequency wavelet coefficients are processed, the average wavelet luminance coefficient $\mathrm{Y}$ is defined, which is shown in Formula (1).

$$
Y=I N T\left(\sum_{k=k_{\min }}^{k=k_{\max }} k p(k)\right) k \in\left[k_{\min }, k_{\max }\right]
$$

In Formula (1), $p(k)$ refers to the probability of wavelet luminance coefficient $k$ that appearing in the low-frequency wavelet coefficients. INT( $\bullet$ ) refers to the rounding function.

Formula (2) describes the histogram equalization of low-frequency wavelet coefficient after the second generation wavelet integer transform of digital image $\mathrm{I}(\mathrm{m}, \mathrm{n})$.

$$
F(i, j)=\operatorname{INT}\left[\left(M_{\max }-M_{\min }\right) \times c d f(f(i, j)) / N+M_{\text {min }}\right]
$$

In Formula (2), $M_{\max }$ and $M_{\min }$ respectively refer to the upper bound and lower bound of the sub-image wavelet luminance coefficients. $\mathrm{N}$ refers to the sum of wavelet coefficients of the low-frequency images. $c d f(f(i, j))$ refers to cumulative function of wavelet coefficients of the low-frequency images. $i, j$ refers to the horizontal and vertical coordinate variables of low frequency images with wavelet three layers decomposition.

The detailed descriptions are shown as follows.

The three layer wavelet decomposition is used for digital image I $(m, n)$ to obtain the third layer low frequency wavelet coefficient matrix and round it to matrix $C A_{3}$;

Use Formula (1) to calculate the average wavelet luminance coefficient $\mathrm{Y}$ of matrix $C A_{3}$;

To calculate the maximum and minimum values $\left(k_{\max }\right.$ and $\left.k_{\min }\right)$ of the integer wavelet coefficient of matrix $\mathrm{CA}_{3}$;

According to the $k_{\max }, k_{\min }$, average wavelet luminance coefficient $\mathrm{Y}$ of matrix $C A_{3}$, we divide matrix $C A_{3}$ into two sub images, $\left[k_{\min }, \mathrm{Y}\right]$ and [Y, $k_{\max }$. Based on Formula (1), sub average wavelet luminance coefficient $Y_{1}$ and $Y_{2}$ of $\left[K_{\min }, \mathrm{Y}\right]$ and $\left[\mathrm{Y}, K_{\max }\right] . \quad Y_{1}=\operatorname{INT}\left(\sum_{k=k_{\min }}^{k=Y} k p(k)\right)$. $Y_{2}=\operatorname{INT}\left(\sum_{k=Y}^{k=k_{\max }} k p(k)\right)$. Finally, matrix $C A_{3}$ is divided into four subinterval images, that is, [ $\left.k_{\min }, Y_{1}\right]$, $\left[Y_{1}, \mathrm{Y}\right],\left[\mathrm{Y}, Y_{2}\right]$ and $\left[Y_{2}, k_{\max }\right]$;

According to Formula (2), the wavelet coefficient histogram equalization of the above four subinterval images is done and merged into the matrix $C A_{3}^{\prime}$;

The reconstruction of low-frequency and high-frequency wavelet coefficients of $C A_{3}^{\prime}$. And the enhanced images are output. 


\section{The Evaluation Indexes of Images}

Average luminance difference $(\Delta Y)$ and Peak Signal-to-Noise Ratio (PSNR) were adopted to objectively analyze the algorithm of this paper, as well as its luminance maintenance and noise controlling. The calculation formulas were as follows.

Average luminance difference $(\Delta Y)$ :

$$
\Delta Y=Y_{F}-Y_{f}=\sum_{k_{F}=0}^{k_{F}=255} k_{F} p\left(k_{F}\right)-\sum_{k_{f}=0}^{k_{f}=255} k_{f} p\left(k_{f}\right)
$$

Mean square error (MSE):

$$
M S E=\frac{1}{m \times n} \sum_{i=1}^{m} \sum_{j=1}^{n}[f(i, j)-\bar{f}(i, j)]^{2}
$$

Peak Signal-to-Noise Ratio (PSNR):

$$
P S N R=10 \lg \left[f^{2}{ }_{\max }(i, j) / M S E\right]
$$

In formula (3), $Y_{F}$ refers to the average luminance of the original image. $Y_{f}$ refers to the enhanced image. $\Delta Y$ is the difference between them. If the value of $\Delta Y$ is less, the luminance of the enhanced image would be much similar to the original one, which showed the better luminance maintenance.

In formula (4) and (5), $\bar{f}(i, j)$ refers to the enhanced image while $f(i, j)$ refers to the original noise image. $m$ and $n$ respectively refers to row and column of the images. If MSE is less and PSNR is larger, the noise controlling was better.

\section{Experimental Simulations and Analysis}

To show the algorithm processing advantages of images with low luminance, this experiment selected the X-ray illumination of foot with low luminance. And the algorithms in literature [2] and literature [8] are compared to the one in this paper. The simulation results are shown in Figure 1.

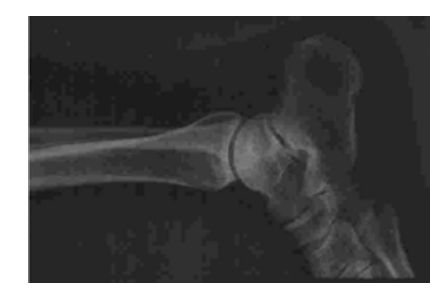

(a) The original image

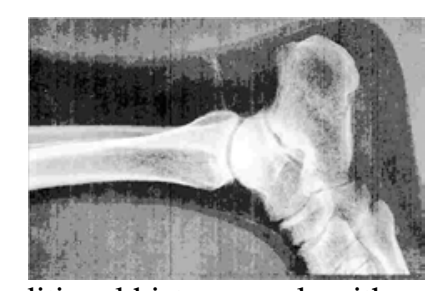

(b) Traditional histogram algorithm

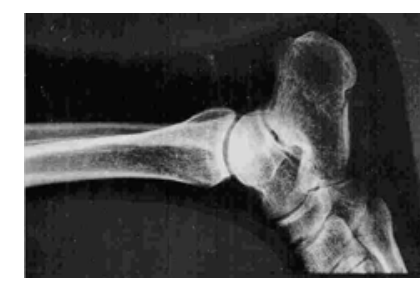

(c) The algorithm in Literature [2]

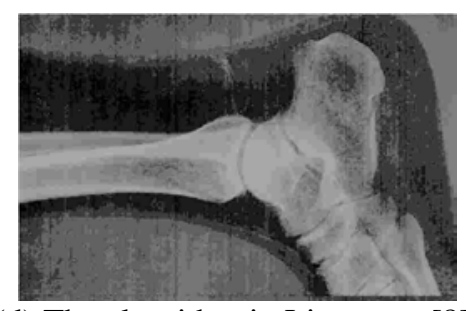

(d) The algorithm in Literature [8]

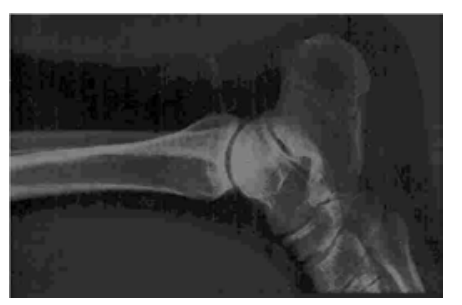

(e) The algorithm in this paper

Figure 1 The experiment results of X-ray medical images

In view of the luminance maintenance of the enhanced images, the images of (b), (c), (d) and (e) in Figure 1 showed that the algorithms in this paper and Literature [2] were better than that in Literature [8] and the traditional one. And the images of (c) and (e) in Figure 1 showed that the algorithm in this paper were better than that in Literature [2].

In view of the details of the enhanced images, the images of (b) and (d) are over improved, which caused the high luminance and unclear details. However, the images of (c) and (e) were relatively better in clarity. While comparing carefully, it was found that the khakud in the images of (c) and (e) 
showed the advantages of algorithm in this paper. That is, the algorithm in this paper helps to keep clearer image details than others.

In the following paper, the noise processing was shown. The standard lena image was chosen. And 0.01 Gaussian noise was added in the original image. The results were shown in Figure 2.

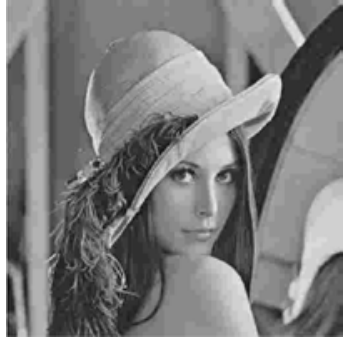

(a)The original image

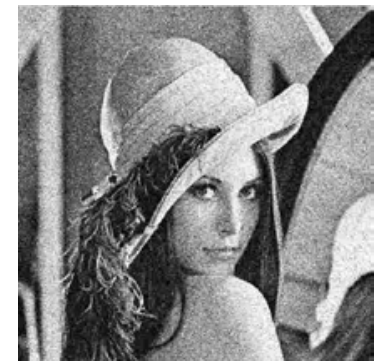

(d) The algorithm in Literature [2]

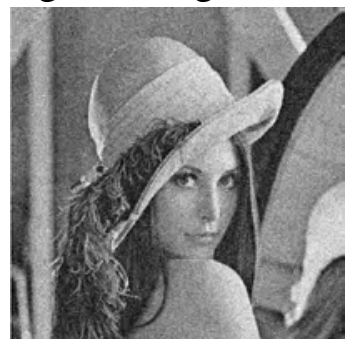

(b) The image with 0.01Gaussian noise

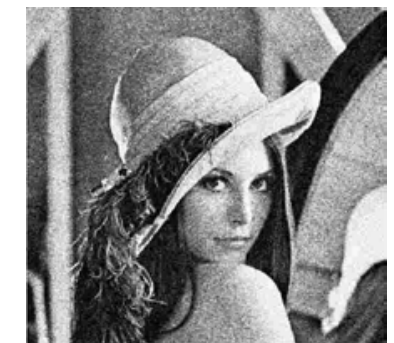

(e) The algorithm in Literature [8]

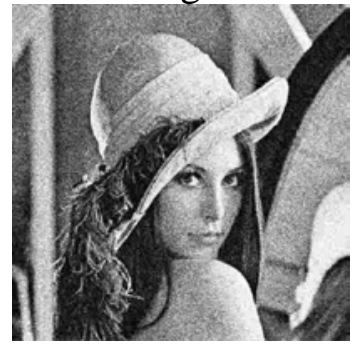

(c) Traditional histogram algorithm

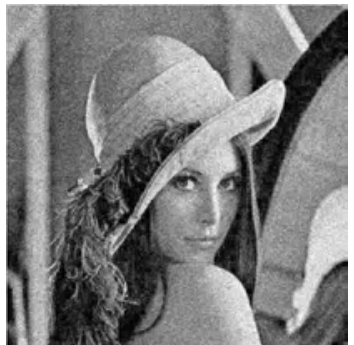

(f) The algorithm in this paper

Figure 2 The experiment results of noise processing

In perspective of noise controlling, the images of (c), (d), (e) and (f) processed by the traditional algorithm and those in Literature [2] and [8] showed that the noise was obviously enlarged. And the image particles were much coarse, which showed the advantage of the algorithm in this paper compared the other three.

The above paper subjectively analyzed the four algorithms in image luminance maintenance and noise controlling. The following paper would subjectively analyze the average luminance difference Peak Signal-to-Noise Ratio (PSNR). The results were shown in Table 1.

Table 1 Calculation results

\begin{tabular}{cccc}
\hline Algorithms & $\begin{array}{c}\text { Mean square } \\
\text { error (MSE) }\end{array}$ & PSNR/dB & $\begin{array}{c}\text { Average } \\
\text { luminance } \\
\text { difference } \\
(\Delta Y)\end{array}$ \\
\hline Traditional histogram algorithm & 97.69 & 28.23 & 9.67 \\
The algorithm in Literature [2] & 102.36 & 28.02 & 7.57 \\
The algorithm in Literature [8] & 63.40 & 30.10 & 12.56 \\
The algorithm in this paper & 84.50 & 28.86 & 4.47 \\
\hline
\end{tabular}

As we can see the values of PSNR in Table 1, the algorithm in Literature [8] got the largest value, followed by the algorithm of this paper. The algorithm in Literature [2] got the lowest value, which was less than the other three. The results showed that the algorithm of this paper was a little poorer than that in Literature [8], but better than those in Literature [2] and the traditional one. The values of average luminance difference $(\Delta Y)$ showed the algorithm of this paper had the lowest value, which proved its advantage in luminance maintenance.

From the above subjective and objective analysis, while processing the images with low luminance, the algorithm of this paper was much better than the other three. 


\section{Conclusions}

In view of the traditional processing defects of histogram image, this paper proposes a new histogram equalization algorithm about second generation wavelet integer transform. The experiment results showed that the algorithm of this paper is better in image luminance maintenance and noise controlling and has clearer details. The visual effects of the enhanced images can be applied in remote sensing satellite and the processing of medical images, etc.

\section{Acknowledgements}

This project is supported by Youth Science Foundation of Jiangsu University of Technology (KYY1407).

\section{References}

[1] ZhANG Guo-dong, YAN Pei-yu \& ZHAO Hong, et al. A contrast enhancement algorithm for low-Dose CT images based on local histogram equalization [C]//Computer and Communication Engineering , International Conference on. Shanghai: IEEE EMBS, 2008: 2462-2465.

[2] Kim Y T. Contrast enhancement using luminance preserving bi-histogram equalization [J]. IEEE Transactions on Consumer Electronics, 1997, 43 (1):1-8.

[3] YANG Yan-chun, DANG Jian-wu \& WANG Yang-ping. A Medical Image Fusion Method Based on Lifting Wavelet Transform and Adaptive PCNN [J]. Journal of Computer-Aided Design \& Computer Graphics, 2012, 24(4): 494-499.

[4] LI Guang-qiong, YU Shao-qian \& CHEN Rong-yuan. Image Enhancement with Adaptive Lifting Wavelet Framework Based on Texture [J]. Computer Applications and Software, 2014, 31(3): 191-194.

[5] Yeganeh H, Ziaei A, Rezaie A. A novel approach for contrast enhancement based on histogram equalization [C]//Computer and Communication Engineering, International Conference on. Kuala Lumpur: IEEE Communications Society, 2008: 256-260.

[6] LENG Lu, LI Ming \& ZHANG Jia-shu. Histogram Equalization Algorithm with Local Adaptive Enhancement Based on Details [J]. Microelectronics \& Computer, 2010, 27(1): 38-41.

[7] HUANG Wei, ZHAO Xiao-ming \& WU Xin-hong, et al. Enhancement Method of Thermal IR Image [J]. Communications Technology, 2009, 42(3): 225-227.

[8] WANG Hai-feng, WU Dong-yan \& ZHANG Yi. Image Enhancement Algorithm of Wavelet Coefficients Equalization about Wavelet Transform [J]. Video Engineering, 2011, 35(19): 27-29. 